\title{
Resolution of pinguecula-related dry eye disease after argon laser photocoagulation
}

This article was published in the following Dove Press journal:

International Medical Case Reports Journal

24 July 2017

Number of times this article has been viewed

\section{Pietro Emanuele Napoli' \\ Raffaele Sanna' \\ Claudio lovino' \\ Maurizio Fossarello,2}

'Department of Surgical Sciences, Eye Clinic, University of Cagliari, Cagliari, Italy; ${ }^{2}$ Clinica Oculistica, San Giovanni di Dio hospital, Azienda Ospedaliera Universitaria di Cagliari, Cagliari, Italy
Correspondence: Pietro Emanuele Napoli Department of Surgical Sciences, University of Cagliari, Via Ospedale 46, 09124 Cagliari, Italy

Tel +393208643697

Email pietronapoli@ymail.com
Purpose: To describe an unusual case of unilateral, chronic symptoms and signs of dry eye, and recurrent episodes of ocular irritation related to pinguecula, which were refractory to prior medical treatments, successfully treated with argon laser photocoagulation.

Methods: A 36-year-old man presented with an elevated yellowish mass on his conjunctiva in his left eye, which was associated with dry eye symptoms and general discomfort, such as "tightening sensation" and "stiffness". On clinical assessment, the left eye revealed a mild conjunctival injection, a fluorescein break-up time (FBUT) of $3.5 \mathrm{~s}$, an abnormal fluorescein staining, and a Schirmer $I$ test of $5 \mathrm{~mm}$. Optical coherence tomography (OCT) imaging evidenced a height at the most elevated point of the pinguecula of $740 \mu \mathrm{m}$. The patient underwent argon-laser photocoagulation.

Results: After 2 weeks postoperatively, no residual of pinguecula or focal injection of the conjunctiva was detected by slit-lamp biomicroscopy and there was a significant improvement of clinical symptoms and signs: Schirmer $I$ test and FBUT increased to $12 \mathrm{~mm}$ and $>10 \mathrm{~s}$, respectively, and fluorescein staining was absent. OCT imaging revealed a smooth structure of conjunctival contour, with a height of $404 \mu \mathrm{m}$. According to an ex juvantibus line of reasoning, definitive diagnosis was pinguecula-related dry eye disease.

Conclusion: Based on the concept that tear film instability and inflammation are two key components of the pathogenesis of dry eye, we coined the term "pinguecula-related dry eye disease," which can be successfully treated by a complete, accurate removal of the lesion with argon laser photocoagulation.

Keywords: pinguecula, tear film instability, argon laser photocoagulation, reflex tear response, ocular surface inflammation

\section{Introduction}

Pingueculae are yellowish, round, elevated masses on the conjunctiva adjacent to the limbus, determining irregularities of the ocular surface contour and, in some cases, resulting in occasional symptoms of discomfort. ${ }^{1}$

Although there is increasing evidence that surface irregularities of the cornea and conjunctiva may lead to tear film instability and add a dry eye component to the ocular surface disorder, and despite tear film instability can induce or exacerbate tear hyperosmolarity (thereby supporting the vicious cycle of dry eye disease), no study has demonstrated dry eye caused by pinguecula. ${ }^{2}$

In fact, some authors have previously suggested the role of the pinguecula merely as a risk factor for dry eye, that is a variable associated with an increased risk of disease. ${ }^{3}$ However, the elimination of a risk factor does not imply the resolution of 
the disease. Risk factors are merely correlational and not necessarily causal. Conversely, in case of cause-and-effect relationship, there would be the resolution of dry eye disease on the elimination of the pinguecula.

Here, we report for the first time a case in which a patient with unilateral, chronic symptoms and signs of dry eye and recurrent episodes of pingueculitis, which were refractory to prior medical treatments (artificial tears and/or topical corticosteroids), was successfully treated with argon laser photocoagulation thus achieving complete remission of both ocular surface disorders.

\section{Case report}

A 36-year-old man was referred to our clinic with an elevated yellowish mass on his conjunctiva in his left eye, which was associated with dry eye symptoms (i.e., soreness, scratchiness, dryness, grittiness, and burning) and general discomfort, such as "tightening sensation" and "stiffness". Interestingly, he reported having chronic inflammation of the aforementioned area for 2-3 years, for which he received topical lubricants and/or steroids, with temporary or partial improvement of ocular irritation.

On clinical assessment, the left eye revealed a yellowwhite, circular bump on the nasal conjunctiva adjacent to the limbus, mild/focal conjunctival injection, a fluorescein break-up time (FBUT) of $3.5 \mathrm{~s}$, an abnormal fluorescein staining (Table 1), and a Schirmer $I$ test of $5 \mathrm{~mm}$. Optical coherence tomography (OCT) imaging evidenced a height at the most elevated point of the pinguecula of $740 \mu \mathrm{m}$ (Figure 1 and Table 1). In contrast, ophthalmic examination of the right eye was unremarkable (Table 1).

McMonnies Dry Eye Questionnaire and Ocular Surface Disease Index (OSDI) were both positive (Table 1). The best-corrected visual acuity (BCVA) was 20/20 in both eyes. Intraocular pressure was $12 \mathrm{mmHg}$ in both eyes. Based on medical history and clinical tests, initial diagnostic hypothesis of pinguecula associated with unilateral dry eye (in the left eye) was made. The diagnostic criteria for dry eye disease and the diagnostic procedures were the same as those used in previous studies. ${ }^{4-6}$

Initially, the patient was again treated with topical lubricant and steroids without any benefit. Thereafter, he underwent argon-laser photocoagulation after being informed of the treatment and its potential complications. An argon green laser (wavelength of $532 \mathrm{~nm}$, Visulas 532s, Carl Zeiss Meditec AG, Jena, Germany) was used for photocoagulation of the lesion by applying the following parameters: duration $0.1 \mathrm{~s}$, power $350-400 \mathrm{~mW}$, and spot size $50 \mu \mathrm{m}$. The procedure was performed after topical instillation of unpreserved $0.4 \%$ oxybuprocaine (Novesina) eye drops. Laser spots were initially targeted to create two grooves perpendicular to each other, and then to cover the entire area of pinguecula with no untreated gaps. Clearly, laser treatment was limited to the lesion and the overlying conjunctiva, sparing surrounding tissues. No discomfort during the laser application was complained of by the patient. The postoperative treatment consisted of an association of betamethasone $0.13 \%$ and chloramphenicol $0.25 \%$ in gel formulation (Betabioptal Gel, Théa Farma SpA) three times a day for 10 days.

In the postoperative period, all ophthalmological examinations were reperformed at the second week, and at the first and third month. At the second week, no residual of pinguecula or focal injection of the conjunctiva was detected by slit-lamp biomicroscopy. The surface contour was flattened as the pinguecula was completely removed, and no defect on the conjunctival epithelium was observed. The BCVA and IOP, respectively, remained $20 / 20$ and $12 \mathrm{mmHg}$ in both eyes. Based on the absence of postoperative dry eye symptoms, McMonnies Dry Eye Questionnaire score and OSDI score, respectively, decreased to three (no symptoms) and zero (no symptoms). Surprisingly, the Schirmer $I$ test increased to $12 \mathrm{~mm}$, and there was a significant improvement in both FBUT (more than $10 \mathrm{~s}$ ) and fluorescein staining (Oxford scheme score was zero). OCT imaging revealed a smooth,

Table I Patient data

\begin{tabular}{|c|c|c|c|c|c|c|}
\hline & $\begin{array}{l}\text { McMonnies } \\
\text { Questionnaire score }\end{array}$ & OSDI score & $\begin{array}{l}\text { Schirmer I test } \\
(\mathrm{mm})\end{array}$ & FBUT (seconds) & $\begin{array}{l}\text { Oxford scheme } \\
\text { score }\end{array}$ & $\begin{array}{l}\text { Height of pinguecula as } \\
\text { assessed by ОСТ }(\mu \mathrm{m})\end{array}$ \\
\hline \multicolumn{7}{|l|}{ Left eye } \\
\hline Baseline & 13 & 15 & 5 & 3.5 & III ( $\leq$ Panel D) & 740 \\
\hline Second week & 3 & 0 & 12 & $>10$ & $0(\leq$ Panel $A)$ & 404 \\
\hline First month & 3 & 0 & 13 & $>10$ & $0(\leq$ Panel $A)$ & 402 \\
\hline Third month & 3 & 0 & 12 & $>10$ & $0(\leq$ Panel $A)$ & 401 \\
\hline \multicolumn{7}{|l|}{ Fellow eye } \\
\hline Baseline & I & 0 & 20 & $>10$ & $0(\leq$ Panel $A)$ & l \\
\hline
\end{tabular}

Notes: Oxford scheme, fluorescein staining of the cornea and conjunctiva graded according to the Oxford system; Height of pinguecula as assessed by OCT, that is, the height of the most elevated point of the lesion from the conjunctival surface.

Abbreviations: FBUT, fluorescein tear break-up time; OCT, optical coherence tomography; OSDI, ocular surface disease index. 


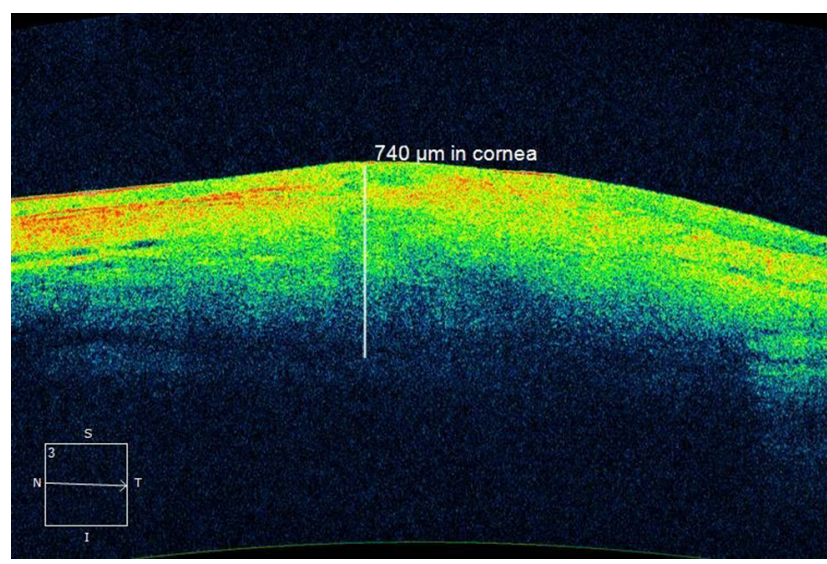

Figure I Optical coherence tomography measurements.

Notes: The location of the apex of the pinguecula was determined by identifying manually the point where there is the most elevated protrusion of conjunctiva. The measurement was made to be perpendicular to the anterior surface of the sclera that is, where there is the interface delineated by differential image reflectivity.

hyper-reflective structure of conjunctival contour, with a height at the most elevated point of $404 \mu \mathrm{m}$. At the subsequent examinations, there were no significant variations (Table 1). Based on response of the dry eye disease to the treatment, and according to an ex juvantibus line of reasoning, definitive diagnosis was pinguecula-related dry eye disease (Figure 2).

Written informed consent to publish this case report and the accompanying images was obtained from the patient.
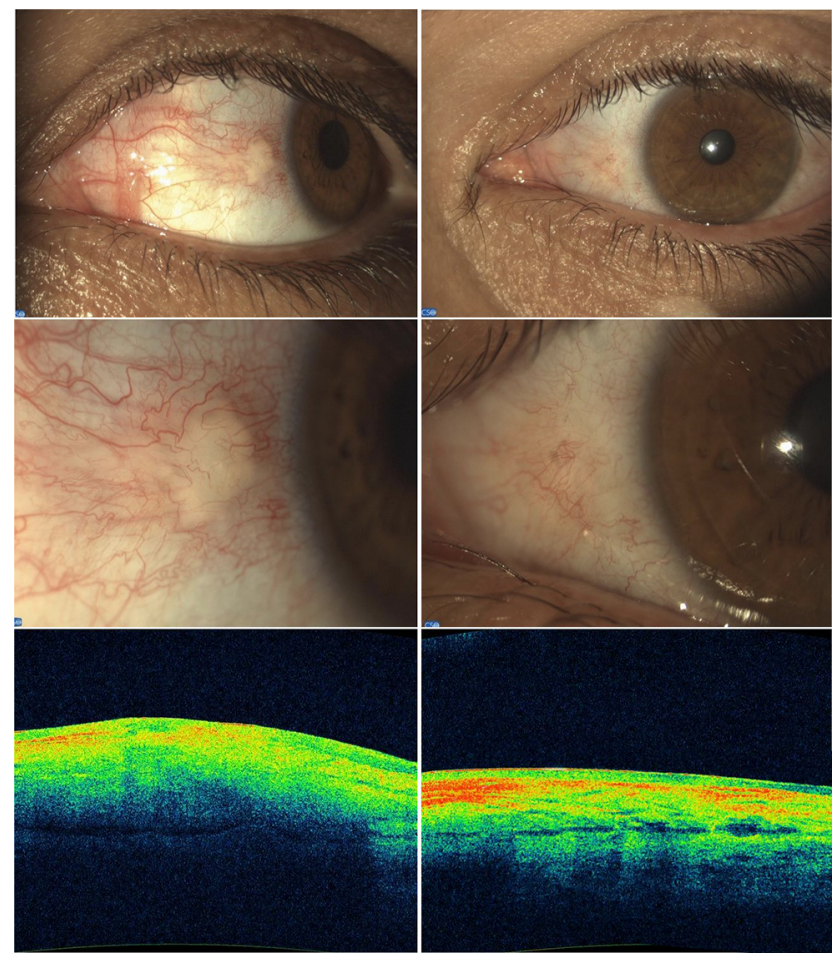

Figure 2 Ocular surface before and after treatment.

Notes: (On the left) Before laser photoablation, pinguecula appeared as an elevated mass on the nasal conjunctiva adjacent to the limbus. (On the right) Three months after photoablation, no residual of pinguecula was detected. (At the bottom) optical coherence tomography images show the changes pre- and post-treatment of the ocular surface contour.

\section{Discussion}

The results of our case report demonstrate, for the first time, that pinguecula may lead to dry eye disease and, in this case, its treatment with photocoagulation may completely resolve the signs and the symptoms of the ocular surface disorder. Clearly, a minimally invasive technique that permits a complete removal of the pinguecula, sparing the surrounding tissue, and a minimal, postoperative inflammatory response are all important aspects to achieve a similar outcome. In addition, laser photoablation permits a good control of tissue ablation in both depth and extent than traditional surgery (spot size is adjustable in microns); it is potentially repeatable and does not require local anesthesia or aseptic procedures. Again, serious conjunctival bleeding (not infrequent after traditional surgery) may be prevented by the coagulation effect of the laser, with benefit for patients with coagulopathy or history of conjunctival surgery.

Interestingly, although it is known that the two eyes usually secrete tears equally (or almost equally), in our case report dry eye syndrome with low tear secretion was unilateral on the same side of the lesion. ${ }^{7}$ However, as previously reported, a reduced tear volume (Schirmer $I$ test $<10 \mathrm{~mm}$ ) is not necessarily associated with pinguecula. ${ }^{8}$ Consequently, a very protuberant pinguecula (in our case, the height was $740 \mu \mathrm{m}$ ), for a long time, may determine such an ocular surface exposure and a poor tear film spreading to induce a marked inflammation of the lacrimal functional unit. In fact, as previously demonstrated, inflammation may, in turn, determine disappearance or dysfunction of cells responsible for tear secretion or retention (chronic secretory dysfunction), by compromising the reflex response and the tear film stability. ${ }^{9}$ It is interesting to note that a reflex tear secretion in response to ocular surface irritation may be only an initial mechanism of compensation. ${ }^{2}$ The chronic surface damage of dry eye may also lead to a decline of reflex tear production and a reduction in corneal sensitivity, thus completing the vicious circle of events. ${ }^{2}$

Based on the concept that tear film instability and inflammation are two key components of the pathogenesis of dry eye, we coined the term "pinguecula-related dry eye disease", the diagnosis of which was made ex juvantibus (based on the resolution after laser treatment), and in which the pinguecula is implicitly the causal agent for dry eye. Again, our case report shows that pinguecula-related dry eye disease may be a primary form of dry eye, unrelated to prior disorders of lid aperture and lid/globe congruity or dynamic.

Although this is the first case in literature that suggests the potential role of pinguecula as a causal agent for dry eye (cause-and-effect relationship) and not merely as a risk factor 
(correlational relationship), our results should be confirmed and analyzed in clinical trials with an adequate sample size. In this sense, it would be interesting to find out how to distinguish the two types of relationship (causal or correlational).

Clearly, the novel approach introduced by us in the present paper represents, at least for now, an experimental, off-label therapy and it should be considered only for patients in whom the first line of medical treatment proved to be ineffective.

In conclusion, here we reported that a pronounced irregularity of the ocular surface due to pinguecula, over time, may determine dry eye disease, which can be successfully treated by a complete, accurate removal of the lesion with argon laser photocoagulation.

\section{Acknowledgment}

The authors would like to acknowledge Luigi Ventura for proofreading the article.

\section{Disclosure}

The authors report no conflicts of interest in this work.

\section{References}

1. Kaji Y, Oshika T, Amano S, Okamoto F, Koito W, Horiuchi S. Immunohistochemical localization of advanced glycation end products in pinguecula. Graefes Arch Clin Exp Ophthalmol. 2006;244(1):104-108.

2. Lemp MA, Foulks GN. The definition and classification of dry eye disease. Ocul Surf. 2007;5(2):75-92.

3. Yokoi N, Tsutomu I, Shigeru K. Surgery of the conjunctiva. Dev Ophthalmol. 2008;41:138-158.

4. Napoli PE, Coronella F, Satta GM, Zucca IA, Fossarello M. A novel OCT technique to measure in vivo the corneal adhesiveness for sodium carboxymethylcellulose in humans and its validity in the diagnosis of dry eye. Invest Ophthalmol Vis Sci. 2014;55(5):3179-3185.

5. Napoli PE, Satta GM, Coronella F, Fossarello M. Spectral-domain optical coherence tomography study on dynamic changes of human tears after instillation of artificial tears. Invest Ophthalmol Vis Sci. 2014;55(7):4533-4540.

6. Napoli PE, Coronella F, Satta GM, Iovino C, Sanna R, Fossarello M. A simple novel technique of infrared meibography by means of spectraldomain optical coherence tomography: a cross-sectional clinical study. PLoS One. 2016;11(10): 0165558.

7. Milder B. The Lacrimal Apparatus in Adler's Physiology of the Eye. 1987.

8. Ahn SJ, Shin KH, Kim MK, Wee WR, Kwon JW. One-year outcome of argon laser photocoagulation of pinguecula. Cornea. 2013;32(7): 971-975.

9. Niederkorn JY, Stern ME, Pflugfelder SC, et al. Desiccating stress induces T cell-mediated Sjögren's syndrome-like lacrimal keratoconjunctivitis. J Immunol. 2006;176(7):3950-3957.
International Medical Case Reports Journal

\section{Publish your work in this journal}

The International Medical Case Reports Journal is an international, peer-reviewed open-access journal publishing original case reports from all medical specialties. Previously unpublished medical posters are also accepted relating to any area of clinical or preclinical science. Submissions should not normally exceed 2,000 words or
Dovepress

4 published pages including figures, diagrams and references. The manuscript management system is completely online and includes a very quick and fair peer-review system, which is all easy to use. Visit $\mathrm{http}: / /$ www.dovepress.com/testimonials.php to read real quotes from published authors.

Submit your manuscript here: https://www.dovepress.com/international-medical-case-reports-journal-journal 